\title{
Formality of Sinha's cosimplicial model for long knots spaces and the Gerstenhaber algebra structure of homology
}

\author{
Paul Arnaud Songhafouo Tsopméné
}

\begin{abstract}
Sinha constructed a cosimplicial space $\mathcal{K}_{N}^{\bullet}$ that gives a model for the space of long knots modulo immersions in $\mathbb{R}^{N}, N \geq 4$. On the other hand, Lambrechts, Turchin and Volić showed that for $N \geq 4$ the homology Bousfield-Kan spectral sequence associated to Sinha's cosimplicial space $\mathcal{K}_{N}^{\bullet}$ collapses at the $E^{2}$ page rationally. Their approach consists in first proving the formality of some other diagrams approximating $\mathcal{K}_{N}^{\bullet}$ and next deducing the collapsing result. In this paper, we prove directly the formality of Sinha's cosimplicial space, which immediately implies the collapsing result for $N \geq 3$. Moreover, we prove that the isomorphism between the $E^{2}$ page and the homology of the space of long knots modulo immersions respects the Gerstenhaber algebra structure, when $N \geq 4$.
\end{abstract}

57Q45, 57Q45; 18D50, 55P48, 17B63

\section{Introduction}

A multiplicative operad in a symmetric monoidal category $\mathcal{C}$ consists of a couple $(\mathcal{O}, \alpha)$ in which $\mathcal{O}$ is a nonsymmetric operad in $\mathcal{C}$ and $\alpha$ is a morphism from the associative operad $\mathcal{A} s$ (see Example 2.1 below for its definition) to $\mathcal{O}$. The multiplicative operad we look at in this paper is Kontsevich's operad $\mathcal{K}_{N}=\left\{\mathcal{K}_{N}(n)\right\}_{n \geq 0}$, which was defined and studied by Sinha in [17, Definition 4.1 and Theorem 4.5]. Notice that this operad is equivalent to the little $N$-disks operad. Notice also that to any multiplicative operad $\mathcal{O}$, one can associate a cosimplicial object $\mathcal{O}^{\bullet}$ (see McClure and Smith [11, Section 10]). Hence the multiplicative operad $\mathcal{K}_{N}$ induces a cosimplicial space, $\mathcal{K}_{N}^{\bullet}$, called Sinha's cosimplicial space.

For $N \geq 3$, a long knot is a smooth embedding $\mathbb{R} \hookrightarrow \mathbb{R}^{N}$ that coincides outside a compact set with a fixed linear embedding. The space of long knots modulo immersion, denoted by $\overline{\mathrm{Emb}}\left(\mathbb{R}, \mathbb{R}^{N}\right)$, is defined as the homotopy fiber of the inclusion of the space of long knots in the space of long immersions. In [17] Sinha proves that for $N \geq 4$, the homotopy totalization of $\mathcal{K}_{N}^{\bullet}$ is weakly equivalent to the space of long knots modulo immersion,

$$
\operatorname{hoTot} \mathcal{K}_{N}^{\bullet} \simeq \overline{\operatorname{Emb}}\left(\mathbb{R}, \mathbb{R}^{N}\right) .
$$


He also proves that for $N \geq 4$, the homology Bousfield-Kan spectral sequence associated to $\mathcal{K}_{N}^{\bullet}$ converges to the homology $H_{*}\left(\right.$ hoTot $\left.\mathcal{K}_{N}^{\bullet}\right) \cong H_{*}\left(\overline{\operatorname{Emb}}\left(\mathbb{R}, \mathbb{R}^{N}\right)\right)[17$, Theorem 7.2]. Therefore it is natural to ask whether this spectral sequence collapses or not. This question was studied by Lambrechts, Turchin and Volić in [8], who proved the following result.

Theorem 1.1 [8, Theorem 1.2] For $N \geq 3$ the homology Bousfield-Kan spectral sequence associated to Sinha's cosimplicial space $\mathcal{K}_{N}^{\bullet}$ collapses at the $E^{2}$ page rationally.

Remark 1.2 Actually in [8] Theorem 1.1 is proved only for $N>3$, but our approach also does the work for $N=3$.

The authors of [8] prove Theorem 1.1 without showing that $\mathcal{K}_{N}^{\bullet}$ is formal. The main ingredient of their proof is the relative version of Kontsevich's theorem on the formality of the little $N$-disks operad; see Lambrechts and Volić [9]. This theorem states that there exists a chain of quasi-isomorphisms of operads between the singular chains on the little $N$-disks operad and its homology with real coefficients. In particular, there is a zigzag

$$
S_{*}\left(\mathcal{K}_{N}\right) \stackrel{\sim}{\longleftarrow} \cdots \stackrel{\sim}{\longrightarrow} H_{*}\left(\mathcal{K}_{N}\right)
$$

in which the chain complex of the Fulton-MacPherson operad $S_{*}\left(\mathcal{F}_{N}\right)$ appears. This poses a serious problem to the authors of [8] because the operad $S_{*}\left(\mathcal{F}_{N}\right)$ is not multiplicative, but only multiplicative "up to homotopy" in the sense of Definition 2.6. This problem is solved by introducing certain finite diagrams of spaces called fanic diagrams. Here are our results.

Theorem 1.3 For $N \geq 3$, the operads $S_{*}\left(\mathcal{K}_{N} ; \mathbb{R}\right)$ and $H_{*}\left(\mathcal{K}_{N} ; \mathbb{R}\right)$ are weakly equivalent as multiplicative operads.

For the meaning of "weakly equivalent as multiplicative operads", see Definition 2.6.

Remark 1.4 In [9] it is only proved that $S_{*}\left(\mathcal{K}_{N} ; \mathbb{R}\right)$ and $H_{*}\left(\mathcal{K}_{N} ; \mathbb{R}\right)$ are weakly equivalent as "up to homotopy multiplicative operads" (Definition 2.6), when $N \geq 3$. Notice that this result is not proved for $N=2$ but only for $N \geq 3$ (see [9, Theorem 1.4]).

An immediate consequence of Theorem 1.3 is the following formality result.

Corollary 1.5 For $N \geq 3$ Sinha's cosimplicial space $\mathcal{K}_{N}^{\bullet}$ is formal over $\mathbb{R}$.

Our method enables us also to determine the Gerstenhaber structure on the homology of the space of long knots. 
We explain now with which Gerstenhaber structures we endow $H_{*}\left(\overline{\operatorname{Emb}}\left(\mathbb{R}, \mathbb{R}^{N}\right) ; \mathbb{R}\right)$ and $H H\left(H_{*} \mathcal{K}_{N} ; \mathbb{R}\right)$.

McClure and Smith construct in [11] two operads, $\mathcal{D}_{2}$ and $\widetilde{\mathcal{D}}_{2}$, both weakly equivalent to the little 2-disks operad $\mathcal{C}_{2}$. They show if a cosimplicial space $\mathcal{O}^{\bullet}$ is built from a multiplicative operad $\mathcal{O}$, then $\mathcal{D}_{2}$ acts on the totalization $\operatorname{Tot} \mathcal{O}^{\bullet}$ [11, Theorem 9.1], and $\widetilde{\mathcal{D}}_{2}$ acts on the homotopy totalization $\operatorname{hoTot} \mathcal{O}^{\bullet}[11$, Theorem 15.3]. If in addition $\mathcal{O}$ is reduced (that is, both $\mathcal{O}(0)$ and $\mathcal{O}(1)$ are weakly contractible), then the homotopy totalization of $\mathcal{O}^{\bullet}$ is weakly homotopy equivalent to the double loop space of a certain explicit space of maps of operads (Dwyer and Hess [3] and Turchin [19] prove this result by using different approaches). Notice that neither Dwyer and Hess nor Turchin actually prove that their delooping is the delooping with respect to the McClure-Smith $\widetilde{\mathcal{D}}_{2}$ action.

We come back now to the particular case of Kontsevich's operad $\mathcal{K}_{N}$, which is reduced. Since it is multiplicative, it follows the operad $\widetilde{\mathcal{D}}_{2}$ acts on hoTot $\mathcal{K}_{N}^{\bullet} \simeq \overline{\operatorname{Emb}}\left(\mathbb{R}, \mathbb{R}^{N}\right)$. We also have a $\mathcal{C}_{2}$ geometric action (constructed by Budney in [2]) on (framed) long knots. One question arises: are these two actions equivalent? This question is still open to my knowledge. But one thing is certain: each of these actions induces a Gerstenhaber algebra structure on $H_{*}\left(\overline{\mathrm{Emb}}\left(\mathbb{R}, \mathbb{R}^{N}\right) ; \mathbb{R}\right)$, and apparently it has never been checked whether these structures coincide. We now specify which one we choose.

(A) The left hand side of equation (1) is equipped with the Gerstenhaber algebra structure induced by the action of $\widetilde{\mathcal{D}}_{2}$ on hoTot $\mathcal{K}_{N}^{\bullet} \simeq \overline{\mathrm{Emb}}\left(\mathbb{R}, \mathbb{R}^{N}\right)$.

On the other hand, associated to a multiplicative operad $\mathcal{B}_{*}(\bullet)$ in chain complexes is its Hochschild homology $H H\left(\mathcal{B}_{*}(\bullet)\right)$, defined first by Gerstenhaber and Voronov in [5]. It is endowed with a natural Gerstenhaber algebra structure (see [5] or Salvatore [16, Section 4] for more details about this natural structure).

(B) The right hand side of equation (1), $H H\left(H_{*} \mathcal{K}_{N} ; \mathbb{R}\right)$, is equipped with the natural Gerstenhaber algebra structure.

Corollary 1.6 For $N \geq 4$, there exists an isomorphism of Gerstenhaber algebras between the homology of space of long knots modulo immersions and the Hochschild homology $H H\left(H_{*} \mathcal{K}_{N}\right)$ over $\mathbb{R}$ when the homology $H_{*}\left(\overline{\mathrm{Emb}}\left(\mathbb{R}, \mathbb{R}^{N}\right) ; \mathbb{R}\right)$ is equipped with the Gerstenhaber algebra structure described by $(A)$, and $H H\left(H_{*} \mathcal{K}_{N} ; \mathbb{R}\right)$ is equipped with the one described by (B). That is,

$$
H_{*}\left(\overline{\operatorname{Emb}}\left(\mathbb{R}, \mathbb{R}^{N}\right) ; \mathbb{R}\right) \cong H H\left(H_{*} \mathcal{K}_{N} ; \mathbb{R}\right) .
$$


Remark 1.7 For $N \geq 4$, it is proved that $H H\left(H_{*} \mathcal{K}_{N} ; \mathbb{Q}\right)$ and $H_{*}\left(\overline{\operatorname{Emb}}\left(\mathbb{R}, \mathbb{R}^{N}\right) ; \mathbb{Q}\right)$ are isomorphic as vector spaces but not as Gerstenhaber algebras [8].

Remark 1.8 In [15, Theorem 2.3] Sakai proves a result, announced earlier by Salvatore [16, Proposition 22], which states that the graded vector spaces $H H\left(S_{*} \mathcal{K}_{N}\right)$ and $H_{*}\left(\overline{\mathrm{Emb}}\left(\mathbb{R}, \mathbb{R}^{N}\right)\right)$ are isomorphic as Gerstenhaber algebras.

Remark 1.9 When a version of this paper was ready, Syunji Moriya put in arXiv a paper [13] in which equivalent results are independently discovered.

Outline of the paper In Section 2 we first recall the definition of a nonsymmetric operad. Next we show that the axiom of relative properness holds in the category of nonsymmetric operads (this is just the nonsymmetric version of Fresse [4, Theorem 12.2.B]). Finally we prove Lemma 2.7, which is crucial for the rest of the paper.

In Section 3 we apply Lemma 2.7 to the specific zigzag between $S_{*}\left(\mathcal{K}_{N} ; \mathbb{R}\right)$ and its homology operad $H_{*}\left(\mathcal{K}_{N} ; \mathbb{R}\right)$, and we obtain the main result of the paper, which states that the Kontsevich's operad is formal over $\mathbb{R}$ as a multiplicative operad (Theorem 1.3). This result implies immediately that Sinha's cosimplicial space is formal over $\mathbb{R}$ (Corollary 1.5). Using now this formality, we give a very short proof of the collapse of the Vassiliev spectral sequence over rationals (Theorem 1.1). We end the section with a proof of Corollary 1.6 which is also a consequence of Theorem 1.3.

Acknowledgements The author is grateful to Pascal Lambrechts for suggesting the idea of left properness axiom and also for his encouragement. I also thank Yves Félix for giving me a copy of [4], which is a central reference for this paper. Obviously, I cannot forget to thank Benoit Fresse and Paolo Salvatore for answering all my emails with questions about the homotopy theory of operads and of cosimplicial spaces.

\section{The category of nonsymmetric operads and equivalences of multiplicative operads}

Throughout this section we denote by $\mathcal{C}:=(\mathcal{C}, \otimes, \mathbf{1})$ a symmetric monoidal model category that is cofibrantly generated [4, Section 11.1.5 and Section 11.3.3]. The category $\mathcal{C}^{\mathbb{N}}$ of sequences $X=\{X(n)\}_{n \geq 0}$ in $\mathcal{C}$ will be equipped with the obvious model structure (that is, weak equivalences, fibrations and cofibrations are all levelwise). Note that model categories for us are as in Hovey [6]. 
We begin by recalling the definition of a nonsymmetric operad. Next we state and prove the nonsymmetric version of [4, Theorem 12.2.B], which states that the axiom of relative properness holds in the category of nonsymmetric operads in $\mathcal{C}$. We end with the crucial Lemma 2.7 for this paper.

A symmetric operad in $\mathcal{C}$ consists of a symmetric sequence $\mathcal{O}=\{\mathcal{O}(n)\}_{n \geq 0}$ (for each $n$ the symmetric group $\Sigma_{n}$ acts on $\mathcal{O}_{n}$ ) of objects of $\mathcal{C}$ endowed with an unit element $\mathbf{1} \longrightarrow \mathcal{O}(1)$ and a collection of morphisms

$$
\mathcal{O}(k) \otimes \mathcal{O}\left(i_{1}\right) \otimes \cdots \otimes \mathcal{O}\left(i_{k}\right) \longrightarrow \mathcal{O}\left(i_{1}+\cdots+i_{k}\right)
$$

that satisfy natural equivariance properties, unit and associative axioms (May's axioms; see [10]). If we omit the action of $\Sigma_{n}$, then $\mathcal{O}$ is called a nonsymmetric operad. The category of nonsymmetric operads in $\mathcal{C}$ will be denoted by $\mathcal{O} p_{n s}(\mathcal{C})$ or by the short notation $\mathcal{O} p_{n s}$.

Example 2.1 Let $\mathcal{A} s=\{\mathcal{A} s(n)\}_{n \geq 0}$ be the sequence defined by $\mathcal{A} s(n)=\mathbf{1}$ for each $n$, the unit for the tensor product of $\mathcal{C}$. It is easy to see that $\mathcal{A} s$ is a nonsymmetric operad, called the associative operad.

Remark 2.2 The object $\mathcal{A} s$ is cofibrant in the category $\mathcal{C}^{\mathbb{N}}$ because the unit object $\mathbf{1}$ is cofibrant in $\mathcal{C}$ by the unit axiom, which is a part of the definition [4, Section 11.3.3] of a symmetric monoidal model category.

Notice that the category $\mathcal{O} p_{n s}(\mathcal{C})$ usually has only semimodel structure, by the nonsymmetric version of [4, Theorem 12.2.A]. But, if $\mathcal{C}$ satisfies certain conditions (see Muro [14, Theorem 1.1]), then $\mathcal{O} p_{n s}(\mathcal{C})$ turns out to be a model category. For example it is not difficult to see that the category of nonnegatively graded chain complexes $\mathrm{Ch}_{\mathbb{R}}$ satisfies such conditions.

Remark 2.3 The category $\mathcal{O} p_{n s}\left(\mathrm{Ch}_{\mathbb{R}}\right)$ is equipped with a model category structure in which weak equivalences and fibrations are all levelwise. Recall that in $\mathrm{Ch}_{\mathbb{R}}$ a morphism is a weak equivalence if it is a quasi-isomorphism, and a fibration if it is an epimorphism.

Let $\mathcal{O} p_{s}(\mathcal{C})$ denote the category of symmetric operads in $\mathcal{C}$. Then the obvious forgetful functor $U: \mathcal{O} p_{s}(\mathcal{C}) \longrightarrow \mathcal{O} p_{n s}(\mathcal{C})$ admits a left adjoint $\operatorname{Sym}: \mathcal{O} p_{n s}(\mathcal{C}) \longrightarrow \mathcal{O} p_{s}(\mathcal{C})$ defined by

$$
\operatorname{Sym}(P)(n)=\Sigma_{n} \otimes P(n)=\coprod_{\sigma \in \Sigma_{n}} P(n) .
$$


The following proposition was first proved by Spitzweck [18] and by Berger and Moerdijk [1] for symmetric operads.

Proposition 2.4 The category of nonsymmetric operads in $\mathcal{C}$ satisfies the axiom of relative properness: If $P$ and $Q$ are objects of $\mathcal{O} p_{n s}$ that are cofibrant in $\mathcal{C}^{\mathbb{N}}$, then the pushout in $\mathcal{O} p_{n s}$ of a weak equivalence along a cofibration

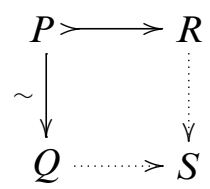

gives a weak equivalence $R \stackrel{\sim}{\longrightarrow} S$.

Proof Since $P$ and $Q$ are cofibrant in the category $\mathcal{C}^{\mathbb{N}}$, it follows that $\operatorname{Sym}(P)$ and $\operatorname{Sym}(Q)$ are $\Sigma_{*}$-cofibrant. By applying the functor Sym to the diagram of the statement, we obtain the following pushout diagram in the category of symmetric operads

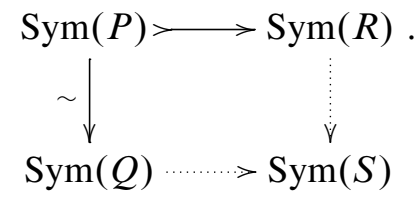

We apply now [4, Theorem 12.2.B] to get a weak equivalence $\operatorname{Sym}(R) \stackrel{\sim}{\longrightarrow} \operatorname{Sym}(S)$. Since $\operatorname{Sym}(R)(n)$ (respectively $\operatorname{Sym}(S)(n))$ is the coproduct over the set $\Sigma_{n}$ of copies of the object $R(n)$ (respectively $S(n)$ ), it follows that the morphism $R \longrightarrow S$ in $\mathcal{O} p_{n s}$ is also a weak equivalence.

Recall now some necessary definitions.

Definition 2.5 - A multiplicative operad in $\mathcal{C}$ is a couple $(\mathcal{O}, \alpha)$ in which $\mathcal{O}$ is a nonsymmetric operad in $\mathcal{C}$ and $\alpha: \mathcal{A} s \longrightarrow \mathcal{O}$ is a morphism of nonsymmetric operads from the associative operad to $\mathcal{O}$.

- An up to homotopy multiplicative operad consists of a triple $(\mathcal{O}, \mathcal{A}, \eta)$ in which $\mathcal{O}$ is a nonsymmetric operad in $\mathcal{C}, \mathcal{A}$ is an operad weakly equivalent to the associative operad $\mathcal{A s}$, and $\eta: \mathcal{A} \longrightarrow \mathcal{O}$ is a morphism of nonsymmetric operads. 
Notice that by Definition 2.5, every multiplicative operad is an up to homotopy multiplicative operad. Therefore the category of multiplicative operads is a full subcategory of the category of up to homotopy multiplicative operads. In the latter category, a morphism from $(\mathcal{O}, \mathcal{A}, \eta)$ to $\left(\mathcal{O}^{\prime}, \mathcal{A}^{\prime}, \eta^{\prime}\right)$ consists of morphisms $g: \mathcal{O} \longrightarrow \mathcal{O}^{\prime}$ and $f: \mathcal{A} \longrightarrow \mathcal{A}^{\prime}$ such that $g \eta=\eta^{\prime} f$.

Definition 2.6 Two multiplicative operads $\mathcal{M}$ and $\mathcal{M}^{\prime}$ are said to be weakly equivalent as multiplicative operads (respectively weakly equivalent as up to homotopy multiplicative operads) if there is a zigzag

$$
\mathcal{M}<\sim \mathcal{O}_{1} \stackrel{\sim}{\longrightarrow} \cdots<\sim \mathcal{O}_{p} \stackrel{\sim}{\longrightarrow} \mathcal{M}^{\prime}
$$

in the category of multiplicative operads (respectively in the category of up to homotopy multiplicative operads).

We are now ready to state and prove our crucial lemma.

Lemma 2.7 In the category $\mathcal{O} p_{n s}$ of nonsymmetric operads in $\mathcal{C}$, consider the commutative diagram

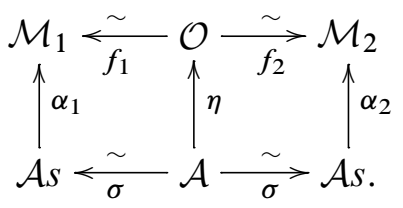

Assume that $\mathcal{A}$ is cofibrant as an object of $\mathcal{C}^{\mathbb{N}}$. Then the operads $\mathcal{M}_{1}$ and $\mathcal{M}_{2}$ are weakly equivalent as multiplicative operads.

Proof We begin with the commutative diagram

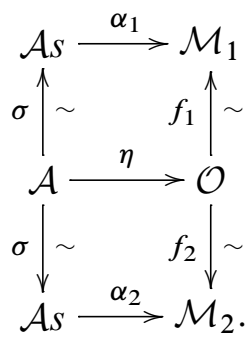


Since the object $\mathcal{A}$ is cofibrant in the category $\mathcal{C}^{\mathbb{N}}$, by applying the factorization axiom to the morphism $\eta: \mathcal{A} \longrightarrow \mathcal{O}$, we obtain the diagram

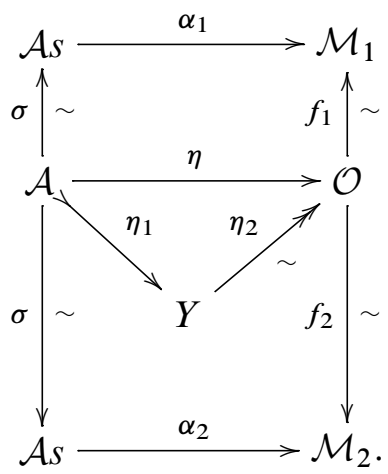

By taking the pushout of the diagram

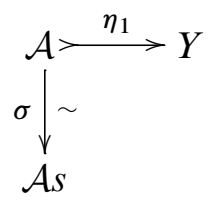

we obtain

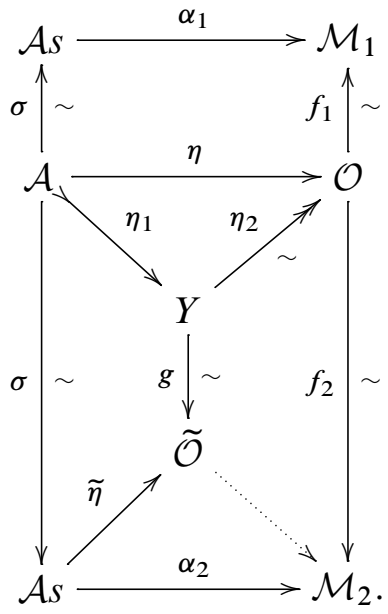

Since the operad $\mathcal{A} s$ is cofibrant in $\mathcal{C}^{\mathbb{N}}$ (see Remark 2.2 above) and $\mathcal{A}$ is also cofibrant in $\mathcal{C}^{\mathbb{N}}$ by hypothesis, and since the morphism $\sigma: \mathcal{A} \longrightarrow \mathcal{A} s$ is a weak equivalence and the morphism $\eta_{1}: \mathcal{A} \longrightarrow Y$ is a cofibration, it follows by Proposition 2.4 that the morphism $g: Y \longrightarrow \widetilde{\mathcal{O}}$ is a weak equivalence. 
Consider now the pushout diagram

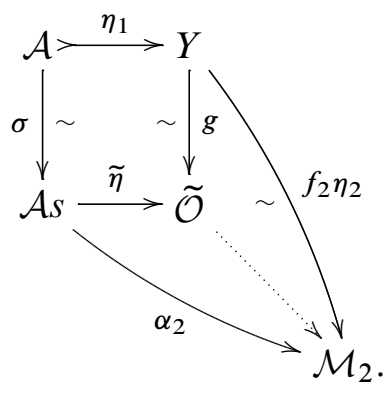

The universal property of the pushout and the two out of three axiom M2 allow us to obtain a weak equivalence

$$
\tilde{f}_{2}: \tilde{\mathcal{O}} \stackrel{\sim}{\longrightarrow} \mathcal{M}_{2}
$$

Similarly, by considering the pushout diagram

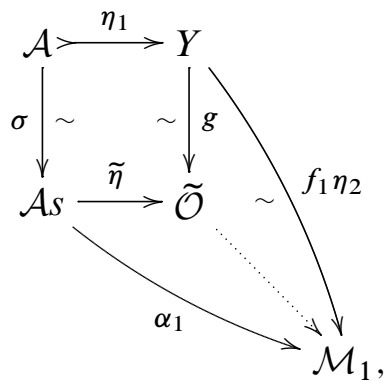

we deduce the existence of a weak equivalence

$$
\tilde{f}_{1}: \widetilde{\mathcal{O}} \stackrel{\sim}{\longrightarrow} \mathcal{M}_{1}
$$

Finally, we obtain the commutative diagram

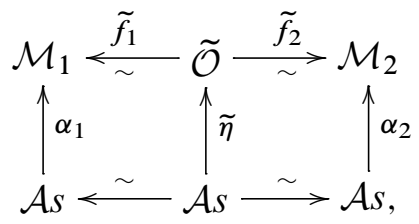

thus completing the proof. 


\section{Formality of the Kontsevich operad as a multiplicative operad}

The goal of this section is to prove Theorem 1.3 and Corollaries 1.5 and 1.6 announced in the introduction. We will also give a very short proof of Theorem 1.1. The ground field in this section is $\mathbb{R}$.

\subsection{Proof of Theorem 1.3}

In [9], P Lambrechts and I Volić develop the details of Kontsevich's proof [7] of the formality of little $N$-disks operad $\mathcal{B}_{N}=\left\{\mathcal{B}_{N}(k)\right\}_{k \geq 0}$ over the real numbers. Note that this formality and its relative version hold in the category $\mathcal{O} p_{n s}\left(\mathrm{Ch}_{\mathbb{R}}\right)$ of nonsymmetric operads in chain complexes over $\mathbb{R}$.

Theorem 3.1 [9, Theorem 1.4] The little $N$-disks operad is relatively formal over the real numbers when $N \geq 3$.

The authors of [9] prove Theorem 3.1 by explicitly constructing a diagram

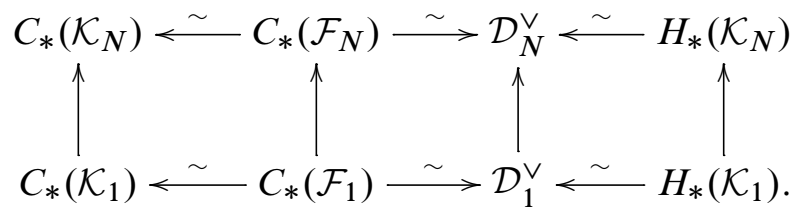

In the previous diagram, the quasi-isomorphism $C_{*}\left(\mathcal{F}_{N}\right) \stackrel{\sim}{\longrightarrow} \mathcal{D} \vee N$ is built in $[9$, Section 9], and the quasi-isomorphism $H_{*}\left(\mathcal{K}_{N}\right) \stackrel{\sim}{\longrightarrow} \mathcal{D}_{N}^{\vee}$ is built in [9, Section 8].

Proof of Theorem 1.3 In the lower row of (2), only $C_{*}\left(\mathcal{F}_{1}\right)$ is not the associative operad. The other three: $C_{*}\left(\mathcal{K}_{1}\right), \mathcal{D}_{1}^{\vee}, H_{*}\left(\mathcal{K}_{1}\right)$ are, and moreover the two morphisms from $C_{*}\left(\mathcal{F}_{1}\right)$ to $\mathcal{A} s$ are the same, and the objects $C_{*}\left(\mathcal{K}_{1}\right)$ and $C_{*}\left(\mathcal{F}_{1}\right)$ are cofibrant in the model category $\mathrm{Ch}_{\mathbb{R}}^{\mathbb{N}}$, which implies the desired result by Lemma 2.7.

\subsection{Proof of Corollary 1.5 and Theorem 1.1}

Recall first that a cosimplicial space $X^{\bullet}$ is said to be formal over $\mathbb{R}$ if the diagram $X^{\bullet}: \Delta \longrightarrow$ Top is formal in the sense that $S_{*}\left(X^{\bullet} ; \mathbb{R}\right)$ and $H_{*}\left(X^{\bullet} ; \mathbb{R}\right)$ are weakly equivalent in the category of cosimplicial chain complexes. 
Proof of Corollary 1.5 and Theorem 1.1 By Theorem 1.3 the operads $S_{*}\left(\mathcal{K}_{N}\right)$ and $H_{*}\left(\mathcal{K}_{N}\right)$ are weakly equivalent as multiplicative operads. Therefore the associated cosimplicial objects $\left(S_{*}\left(\mathcal{K}_{N}\right)\right)^{\bullet}$ and $\left(H_{*}\left(\mathcal{K}_{N}\right)\right)^{\bullet}$ are weakly equivalent in the category of cosimplicial chain complexes over $\mathbb{R}$, hence $S_{*}\left(\mathcal{K}_{N}^{\bullet}\right)$ is formal over $\mathbb{R}$. Now the collapsing of the Bousfield Kan spectral sequence comes from the fact that in the $E^{2}$ page we can replace the column $S_{*}\left(\mathcal{K}_{N}^{p}\right)$ by the homology $H_{*}\left(\mathcal{K}_{N}^{p}\right)$, hence the vertical differential vanishes and the spectral sequence collapses (see [8, Proposition 3.2]).

\subsection{Gerstenhaber algebra structure on the homology of the space of long knots}

The goal here is to prove Corollary 1.6 announced in the introduction. In [12] McClure and Smith construct an $E_{2}$ chain operad $\mathcal{T}_{2}$ that acts on the Hochshild complex $C H\left(B_{*}\right)$, when $B_{*}$ is an operad with multiplication in chain complexes (recall that an $E_{2}$ chain operad is a chain operad weakly equivalent to the normalized singular chain of the little 2-cubes operad). This action induces a Gerstenhaber algebra structure on the Hochshild homology $H H\left(B_{*}\right)$. It is very important to note that this structure coincides with the natural one [5] because $\mathcal{T}_{2}$ is a solution of Deligne's conjecture. Let $\mathcal{T}_{2}$-algebras denote the category of chain complexes equipped with an action of the operad $\mathcal{T}_{2}$. Let $\mathcal{O} p_{*}\left(\mathrm{Ch}_{\mathbb{R}}\right)$ denote the category of multiplicative nonsymmetric operads in chain complexes over real numbers.

Lemma 3.2 [12] There exists a functor

$\mathrm{CH}: \mathcal{O} p_{*}\left(\mathrm{Ch}_{\mathbb{R}}\right) \longrightarrow \mathcal{T}_{2}$-algebras

that preserves weak equivalences.

We are now ready to prove Corollary 1.6.

Proof of Corollary 1.6 First, in the category of multiplicative operads in chain complexes over $\mathbb{R}$, we have by Theorem 1.3 a zigzag

$$
S_{*}\left(\mathcal{K}_{N}\right) \stackrel{\sim}{\longleftarrow} \cdots \stackrel{\sim}{\longrightarrow} H_{*}\left(\mathcal{K}_{N}\right) .
$$

Next, by applying the normalized Hochshild complex functor $\mathrm{CH}$ to (3), we obtain a zigzag

$$
\mathrm{CH}\left(S_{*}\left(\mathcal{K}_{N}\right)\right) \stackrel{\sim}{\sim} \cdots \stackrel{\sim}{\longrightarrow} \mathrm{CH}\left(H_{*}\left(\mathcal{K}_{N}\right)\right)
$$

in the category of $\mathcal{T}_{2}$-algebras by Lemma 3.2. Therefore the homology of (4) gives

$$
H H\left(S_{*}\left(\mathcal{K}_{N}\right)\right) \cong \cdots \stackrel{\cong}{\longrightarrow} H H\left(H_{*}\left(\mathcal{K}_{N}\right)\right),
$$


which respects the Gerstenhaber algebra structure induced by the $H_{*}\left(\mathcal{T}_{2}\right)$ action. Finally, the desired result follows from (5) and Remark 1.8.

\section{References}

[1] C Berger, I Moerdijk, Axiomatic homotopy theory for operads, Comment. Math. Helv. 78 (2003) 805-831 MR2016697

[2] R Budney, Little cubes and long knots, Topology 46 (2007) 1-27 MR2288724

[3] W Dwyer, K Hess, Long knots and maps between operads, Geom. Topol. 16 (2012) 919-955 MR2928985

[4] B Fresse, Modules over operads and functors, Lecture Notes in Mathematics 1967, Springer, Berlin (2009) MR2494775

[5] M Gerstenhaber, A A Voronov, Homotopy G-algebras and moduli space operad, Internat. Math. Res. Notices (1995) 141-153 MR1321701

[6] M Hovey, Model categories, Mathematical Surveys and Monographs 63, American Mathematical Society (1999) MR1650134

[7] M Kontsevich, Operads and motives in deformation quantization, Lett. Math. Phys. 48 (1999) 35-72 MR1718044

[8] P Lambrechts, V Turchin, I Volić, The rational homology of spaces of long knots in codimension > 2, Geom. Topol. 14 (2010) 2151-2187 MR2740644

[9] P Lambrechts, I Volić, Formality of the little N-disks operad (2012) arXiv: $0808.0457 \mathrm{v} 3$

[10] J P May, The geometry of iterated loop spaces, volume 271 of Lectures Notes in Mathematics, Springer, Berlin (1972) MR0420610

[11] J E McClure, J H Smith, Cosimplicial objects and little $n$-cubes, I, Amer. J. Math. 126 (2004) 1109-1153 MR2089084

[12] J E McClure, J H Smith, Operads and cosimplicial objects: an introduction, from: "Axiomatic, enriched and motivic homotopy theory", (J P C Greenlees, editor), NATO Sci. Ser. II Math. Phys. Chem. 131, Kluwer Acad. Publ., Dordrecht (2004) 133-171 MR2061854

[13] S Moriya, Sinha's spectral sequence and homotopical algebra of operads arXiv: 1210.0996

[14] F Muro, Homotopy theory of nonsymmetric operads, Algebr. Geom. Topol. 11 (2011) 1541-1599 MR2821434

[15] K Sakai, Poisson structures on the homology of the space of knots, from: "Groups, homotopy and configuration spaces", (N Iwase, T Kohno, R Levi, D Tamaki, J Wu, editors), Geom. Topol. Monogr. 13 (2008) 463-482 MR2508219 
[16] P Salvatore, Knots, operads, and double loop spaces, Int. Math. Res. Not. 2006 (2006) Art. ID 13628, 22 MR2276349

[17] DP Sinha, Operads and knot spaces, J. Amer. Math. Soc. 19 (2006) 461-486 MR2188133

[18] M Spitzweck, Operads, Algebras and Modules in General Model Categories arXiv: math.AT/0101102

[19] V Turchin, Delooping totalization of a multiplicative operad arXiv:1012.5957

Université catholique de Louvain

Chemin du Cyclotron 2, B-1348 Louvain-la-Neuve, Belgium

arnaud. songhafouo@uclouvain. be

Received: 11 December 2012 Revised: 6 March 2013 